\title{
Limit Cycles and Analytic Centers for a Family of $4 n-1$ Degree Systems with Generalized Nilpotent Singularities
}

\author{
Yusen $\mathrm{Wu},{ }^{1}$ Cui Zhang, ${ }^{2}$ and Changjin $\mathrm{Xu}^{3}$ \\ ${ }^{1}$ School of Mathematics and Statistics, Henan University of Science and Technology, Luoyang, Henan 471023, China \\ ${ }^{2}$ College of Mathematical Science, Luoyang Normal University, Luoyang, Henan 471022, China \\ ${ }^{3}$ Guizhou Key Laboratory of Economics System Simulation, Guizhou University of Finance and Economics, Guiyang, \\ Guizhou 550004, China
}

Correspondence should be addressed to Yusen Wu; wuyusen621@126.com

Received 25 July 2014; Accepted 11 September 2014

Academic Editor: Józef Banaś

Copyright (C) 2015 Yusen Wu et al. This is an open access article distributed under the Creative Commons Attribution License, which permits unrestricted use, distribution, and reproduction in any medium, provided the original work is properly cited.

With the aid of computer algebra system Mathematica 8.0 and by the integral factor method, for a family of generalized nilpotent systems, we first compute the first several quasi-Lyapunov constants, by vanishing them and rigorous proof, and then we get sufficient and necessary conditions under which the systems admit analytic centers at the origin. In addition, we present that seven amplitude limit cycles can be created from the origin. As an example, we give a concrete system with seven limit cycles via parameter perturbations to illustrate our conclusion. An interesting phenomenon is that the exponent parameter $n$ controls the singular point type of the studied system. The main results generalize and improve the previously known results in Pan.

\section{Introduction}

A famous problem for the plane analytic systems of differential equations is under what conditions the local phase portrait at a critical point $p$ is topologically equivalent to the local phase portrait of the linear part of the system at $p$. This problem has been solved by Poincaré and Bendixson for hyperbolic critical points and for elementary critical points, that is, for points having zero determinant and nonzero trace linear part. Another problem is to characterize the local phase portrait at an isolated critical point $p$.

When the matrix of the linear part at the origin is not identically null but has its eigenvalues which are equal to zero, at this moment, the origin is a nilpotent critical point. An analytic system having an isolated nilpotent singularity at the origin, in some suitable coordinates, can be written as follows:

$$
\begin{gathered}
\frac{d x}{d t}=y+\sum_{i+j=2}^{\infty} a_{i j} x^{i} y^{j}=y+X(x, y), \\
\frac{d y}{d t}=\sum_{i+j=2}^{\infty} b_{i j} x^{i} y^{j}=Y(x, y) .
\end{gathered}
$$

Suppose that the function $y=y(x)$ satisfies $X(x, y)=0$, $y(0)=0$. Amelkin et al. proved (see, for instance, [1]) that the origin of system (1) is a monodromic critical point (i.e., a center or a focus) if and only if

$$
\begin{gathered}
Y(x, y(x))=\alpha x^{2 n-1}+o\left(x^{2 n-1}\right), \quad \alpha<0, \\
{\left[\frac{\partial X(x, y)}{\partial x}+\frac{\partial Y(x, y)}{\partial x}\right]_{y=y(x)}=\beta x^{n-1}+o\left(x^{n-1}\right),} \\
\beta^{2}+4 n \alpha<0,
\end{gathered}
$$

where $n$ is a positive integer. Andreev [2] shows what the behavior of the solutions in a neighborhood of the nilpotent critical point is, except if it is a center or a focus (nilpotent center problem). This last result can not distinguish between a focus and a center. Takens [3] and Bogdanov [4] find easy formal normal forms for the nilpotent critical points. Moussu [5] has found the $C^{\infty}$ normal form for a nilpotent center and asks if there exists an analytic normal form for the nilpotent centers. Takens [3] proves that for any analytic nilpotent center there exists an analytic change of variables such that the new system can be written as a system of the 
form (1) and it is a time-reversible nilpotent center. However, the aforementioned result is difficult to implement because, in general, in order to decide if an analytic system has a nilpotent center, we must know explicitly the analytic change of variables which writes system (1) in the Berthier-Moussu normal form. Writing systems in a convenient normal form, Teixeira and Yang [6] study the relationship between timereversibility and the center-focus problem for elementary singular points and nilpotent singular points. Giné [7] develops a method which provides necessary conditions for obtaining a local analytic integral in a neighborhood of a generalized nilpotent singular point. García and Giné [8] give a necessary condition to have local analytic integrability in an analytic nilpotent center. Giacomini et al. [9] studied the centers of planar analytic vector fields which are a limit of linear-type centers.

In this paper, we consider the system of differential equations in the plane whose origin is a generalized nilpotent singular point

$$
\begin{gathered}
\frac{d x}{d t}=y^{2 n-1}+y^{n-1}\left(a_{30} x^{3}+a_{21} x^{2} y^{n}+a_{12} x y^{2 n}+a_{03} y^{3 n}\right) \\
\frac{d y}{d t}=-2 x^{3}+b_{02} y^{2 n}+b_{12} x y^{2 n}+b_{03} y^{3 n}
\end{gathered}
$$

The origin of system (3) is a third-order nilpotent singular point when $n=1$, while it is a total degenerate singular point when $n>1$.

In Section 2, we give some preliminary knowledge concerning the nilpotent critical point. In Section 3, we transform the origin into a third-order nilpotent singular point by a homeomorphism. Then, we compute the first several quasiLyapunov constants and derive the sufficient and necessary conditions for the origin to be an analytic center. In the last section, we prove that there exist seven small amplitude limit cycles in the neighborhood of the origin.

\section{Computation of Quasi-Lyapunov Constants and Determination of Analytic Centers}

In this section, we first introduce some definitions, notations, and symbols in order to make the paper compact and clear, followed by an algorithm to obtain the necessary conditions for the third-order nilpotent critical point of system (1) to be an analytic center, and then we present several methods to prove the sufficiency. More details are due to $[10,11]$.

If the origin of system (1) is a high-order critical point, it is called a $(2 n-1)$ th-order critical point when $(2)$ is satisfied, and it could be broken into $2 n-1$ elementary critical points in the neighborhood of the origin in the complex plane. It is easy to show that the origin of system (1) is a third-order monodromic critical point if and only if $b_{20}=0$ and $\left(2 a_{20}-\right.$ $\left.b_{11}\right)^{2}+8 b_{30}<0$.

Without loss of generality, we assume that

$$
a_{20}=\mu, \quad b_{20}=0, \quad b_{11}=2 \mu, \quad b_{30}=-2 .
$$

Otherwise, by letting $\left(2 a_{20}-b_{11}\right)^{2}+8 b_{30}=-16 \lambda^{2}, 2 a_{20}+$ $b_{11}=4 \lambda \mu$, and taking the transformation $\xi=\lambda x$, $\eta=\lambda y+(1 / 4)\left(2 a_{20}-b_{11}\right) \lambda x^{2}$, we obtain the desired form. Under (4), system (1) becomes the following real autonomous planar system:

$$
\begin{gathered}
\frac{d x}{d t}=y+\mu x^{2}+\sum_{i+2 j=3}^{\infty} a_{i j} x^{i} y^{j}=X(x, y), \\
\frac{d y}{d t}=-2 x^{3}+2 \mu x y+\sum_{i+2 j=4}^{\infty} b_{i j} x^{i} y^{j}=Y(x, y) .
\end{gathered}
$$

In the following, we are going to classify the third-order nilpotent critical point.

Theorem 1. For system (5), there exists a series with nonzero convergence radius:

$$
\begin{gathered}
u(x, y)=x+\sum_{\alpha+\beta=2}^{\infty} a_{\alpha \beta}^{\prime} x^{\alpha} y^{\beta}, \\
v(x, y)=y+\sum_{\alpha+\beta=2}^{\infty} b_{\alpha \beta}^{\prime} x^{\alpha} y^{\beta}, \quad b_{20}^{\prime}=-\mu, \\
\zeta(x, y)=1+\sum_{\alpha+\beta=1}^{\infty} c_{\alpha \beta}^{\prime} x^{\alpha} y^{\beta}
\end{gathered}
$$

such that, by the transformation

$$
u=u(x, y), \quad v=v(x, y), \quad d t=\zeta(x, y) d \tau,
$$

system (5) is reduced to the following Liénard equation:

$$
\begin{gathered}
\frac{d u}{d \tau}=v+\sum_{k=1}^{\infty} A_{k} u^{4 k}+\sum_{k=0}^{\infty} B_{k} u^{4 k+2}+\sum_{k=1}^{\infty} C_{k} u^{2 k+1}=U(u, v) \\
\frac{d v}{d \tau}=-2\left(1+\mu^{2}\right) u^{3}=V(u, v)
\end{gathered}
$$

where $B_{0}=2 \mu$. In addition, the origin of system (5) is a center if and only if $C_{k}=0$ for all $k$.

The following two definitions are taken from [10].

Definition 2. (1) If $\mu \neq 0$, then the origin of system (5) is called a third-order nilpotent critical point of zero-class.

(2) If $\mu=0$ and there exists a positive integer $s$, such that $B_{0}=B_{1}=\cdots=B_{s-1}=0$, but $B_{s} \neq 0$, then the origin of system (5) is called a third-order nilpotent critical point of $s$-class.

(3) If $\mu=0$ and $B_{s}=0$ for all positive integer $s$, then the origin of system (5) is called a third-order nilpotent critical point of $\infty$-class.

Definition 3. Let $f_{k}, g_{k}$ be continuous and bounded functions with respect to $\mu$ and $a_{i j}, b_{i j}, k=1,2, \ldots$ If, for any integer $m$, there exist continuous and bounded functions of $\mu$ and all $a_{i j}, b_{i j}: \xi_{1}^{(m)}, \xi_{2}^{(m)}, \ldots, \xi_{m-1}^{(m)}$, such that

$$
f_{m}=g_{m}+\left(\xi_{1}^{(m)} f_{1}+\xi_{2}^{(m)} f_{2}+\cdots+\xi_{m-1}^{(m)} f_{m-1}\right),
$$


then we say that $f_{m}$ and $g_{m}$ are equivalent, denoted by $f_{m} \sim$ $g_{m}$. If, for any integer $m$, we have $f_{m} \sim g_{m}$, we say that the sequences of functions $\left\{f_{m}\right\}$ and $\left\{g_{m}\right\}$ are equivalent, denoted by $\left\{f_{m}\right\} \sim\left\{g_{m}\right\}$.

Remark 4. It is easy to see from Definition 3 that the following conclusions hold.

(1) The equivalence relationship of two sequences of functions is self-reciprocal, symmetric, and transmissible.

(2) If, for some integer $m, f_{m} \sim g_{m}$, then when $f_{1}=f_{2}=$ $\cdots=f_{m-1}=0$, we have $f_{m}=g_{m}$.

(3) The relationship $f_{1} \sim g_{1}$ implies that $f_{1}=g_{1}$.

The following three theorems were proved in [10].

Theorem 5. If the origin of system (5) is s-class, then the origin of system (5) is a center if and only if there is an inverse integrating factor $M^{s+1}$.

If the origin of system (5) is $\infty$-class, then the origin of system (5) is a center if and only if for any natural number $l$ there exists an inverse integrating factor $M^{l+1}$, where $M$ is a power series given by

$$
M=x^{4}+y^{2}+\sum_{k+2 j=5}^{\infty} c_{k j} x^{k} y^{j}
$$

Theorem 6. For any natural number s and a given number sequence

$$
\left\{c_{0 \beta}\right\}, \quad \beta \geq 3 \text {, }
$$

terms with the coefficients $c_{\alpha \beta}$ satisfying $\alpha \neq 0$ of the formal series (10) can be constructed successively such that

$$
\frac{\partial}{\partial x}\left(\frac{X}{M^{s+1}}\right)+\frac{\partial}{\partial y}\left(\frac{Y}{M^{s+1}}\right)=\frac{1}{M^{s+2}} \sum_{m=6}^{\infty} \omega_{m} x^{m}
$$

that is,

$$
\left(\frac{\partial X}{\partial x}+\frac{\partial Y}{\partial y}\right) M-(s+1)\left(\frac{\partial M}{\partial x} X+\frac{\partial M}{\partial y} Y\right)=\sum_{m=6}^{\infty} \omega_{m} x^{m},
$$

where $s \mu=0$.

Theorem 7. For $\alpha \geq 1, \alpha+\beta \geq 3$ in (11) and (12), $c_{\alpha \beta}$ is uniquely determined by the recursive formula

$$
c_{\alpha \beta}=\frac{1}{(s+1) \alpha}\left(A_{\alpha-1, \beta+1}+B_{\alpha-1, \beta+1}\right) .
$$

For $m \geq 1, \omega_{m}(s, \mu)$ is uniquely determined by the recursive formula

$$
\omega_{m}=A_{m, 0}+B_{m, 0},
$$

where

$$
\begin{aligned}
& A_{\alpha \beta}=\sum_{k+j=2}^{\alpha+\beta-1}[k-(s+1)(\alpha-k+1)] a_{k j} c_{\alpha-k+1, \beta-j}, \\
& B_{\alpha \beta}=\sum_{k+j=2}^{\alpha+\beta-1}[j-(s+1)(\beta-j+1)] b_{k j} c_{\alpha-k, \beta-j+1} .
\end{aligned}
$$

In (15), one sets

$$
\begin{gathered}
c_{00}=c_{10}=c_{01}=0, \\
c_{20}=c_{11}=0, \quad c_{02}=1, \\
c_{\alpha \beta}=0, \quad \text { if } \alpha<0 \text { or } \beta<0 .
\end{gathered}
$$

It follows from Theorems 5-7 that if the origin of system (5) is a center with $s$-class or $\infty$-class, then, by choosing $\left\{c_{0 \beta}\right\}$, such that

$$
\omega_{k}=0, \quad k=6,7, \ldots
$$

The following conclusion holds (see Theorem 3.4 of [10]).

Theorem 8. If the origin of system (5) is $\infty$-class, then when the origin of system (5) is a center, in a neighborhood of the origin, system (5) has an analytic inverse integrating factor

$$
M_{\infty}=1+\text { h.o.t. }
$$

and an analytic first integral given by

$$
F(x, y)=x^{4}+y^{2}+\sum_{k+2 j=5}^{\infty} C_{k j} x^{k} y^{j}
$$

According to [11], we have the following lemma.

Lemma 9. For system (5), if there exists a first integral which is the power series (20) in the neighborhood of the origin, then the origin of system (5) is a center.

By Theorem 1, if the origin of system (5) is a center, there exist analytic transformations in the neighborhood of the origin such that system (5) can be transformed into the Liénard equations:

$$
\begin{aligned}
& \frac{d u}{d \tau}=v+\sum_{k=1}^{\infty} A_{k} u^{4 k}+\sum_{k=0}^{\infty} B_{k} u^{4 k+2}, \\
& \frac{d v}{d \tau}=-2\left(1+\mu^{2}\right) u^{3}, \quad B_{0}=2 \mu .
\end{aligned}
$$

The vector field defined in (21) is symmetrical with respect to the $v$-axis. Further, by the transformation

$$
w=u^{2}, \quad v=v, \quad d \tau=-\frac{d \tau^{\prime}}{2 u},
$$

system (21) is reduced to

$$
\begin{gathered}
\frac{d w}{d \tau^{\prime}}=-v-\sum_{k=1}^{\infty} A_{k} w^{2 k}-\sum_{k=0}^{\infty} B_{k} w^{2 k+1}, \\
\frac{d v}{d \tau^{\prime}}=\left(1+\mu^{2}\right) w, \quad B_{0}=2 \mu .
\end{gathered}
$$


In the $(w, v)$-phase plane, the origin of system (23) is an elementary critical point (focus or center). Obviously, we have the following.

Lemma 10. If there exists a power series $\mathscr{F}=\mathscr{F}(u, v)$ in $u, v$ satisfying

$$
\left.\frac{d \mathscr{F}}{d \tau}\right|_{(21)}=0
$$

then $\mathscr{F}$ can be written as a power series in $u^{2}, v$; namely, $\mathscr{F}(u, v)=G\left(u^{2}, v\right)$.

Then, we have the following theorem.

Theorem 11. The origin of system (5) is an analytic center if and only if the origin of system (5) is a center and $\infty$-class; namely, the origin of system (5) is a center and for any natural number $k, B_{k}=0$.

Corollary 12. If $\mu \neq 0$, the origin of system (5) is not an analytic center.

Theorem 13. The origin of system (5) is an analytic center if and only if there exists an analytic first integral $F(x, y)$ in the neighborhood of the origin, which is the power series (23).

From Theorems 8 and 13, we further have the following.

Theorem 14. The origin of system (5) is an analytic center if and only if in the neighborhood of the origin of system (5) there exists an analytic inverse integrating factor

$$
M_{\infty}=1+\sum_{k+j=1}^{\infty} C_{k j}^{\prime} x^{k} y^{j}
$$

Similarly, by Theorems 5 and 11, we have the following.

Theorem 15. The origin of system (5) is an analytic center, if and only if, for any natural number s, there exists an inverse integrating factor $M^{s+1}$, where $M$ is the power series (10).

Moreover, Theorems 7 and 15 imply the following.

Theorem 16. The origin of system (5) is an analytic center, if and only if, for any natural number s, there exists a power series $M$ satisfying $\omega_{m}=0$ in (12) for all $m$.

Therefore, a new method of determining analytic nilpotent center for a given system has been obtained in Theorem 16.

Theorem 17. If the origin of system (5) is a nilpotent center and system (5) is symmetric with respect to the origin, namely,

$$
\begin{aligned}
& X(-x,-y)=-X(x, y), \\
& Y(-x,-y)=-Y(x, y),
\end{aligned}
$$

then the origin of system (5) is an analytic center.
Theorem 18. If system (5) is symmetric with respect to the $x$ axis, then the origin of system (5) is an analytic center.

Remark 19. If system (5) is symmetric with respect to the $y$ axis, then the origin of system (5) may not be an analytic center. For example, system (21) is symmetric with respect to the $v$-axis, but the origin is an analytic center if and only if $B_{k}=0$ for all $k$.

Eventually, by Theorem 11, we have the following.

Theorem 20. The origin of system (5) is an analytic center if and only if system (5) can be changed into

$$
\frac{d u}{d \tau}=v+\sum_{k=1}^{\infty} A_{k} u^{4 k}, \quad \frac{d v}{d \tau}=-2 u^{3}
$$

by the analytic transformation (7).

\section{Analytic Center Conditions}

In this section we will derive conditions for the origin of system (3) to be an analytic center.

After the change

$$
x_{1}=x, \quad y_{1}=\frac{1}{\sqrt{n}} y^{n}, \quad d t_{1}=\sqrt{n} y^{n-1} d t
$$

and renaming $\left(x_{1}, y_{1}, t_{1}\right)$ with $(x, y, t)$, system (3) takes the form

$$
\begin{gathered}
\frac{d x}{d t}=y+\frac{1}{\sqrt{n}} a_{30} x^{3}+a_{21} x^{2} y+\sqrt{n} a_{12} x y^{2}+n a_{03} y^{3}, \\
\frac{d y}{d t}=-2 x^{3}+n b_{02} y^{2}+n b_{12} x y^{2}+n^{3 / 2} b_{03} y^{3} .
\end{gathered}
$$

According to Theorem 7, we have the following.

Lemma 21. Assume that $s$ is a natural number. One can derive a power series (10) for system (29) under which (12) is satisfied, where

$$
\begin{array}{clrl}
c_{00}=0, & c_{10}=0, & c_{01}=0, & c_{20}=0, \\
c_{11}=0, & c_{02}=1 .
\end{array}
$$

In addition, for any natural numbers $\alpha, \beta, c_{\alpha \beta}$ is given by the following recursive formula:

$$
\begin{aligned}
c_{\alpha \beta}=(2 & (1+s)(2+\beta) c_{-4+\alpha, 2+\beta} \\
& +\frac{1}{\sqrt{n}} a_{30}(3-(1+s)(-3+\alpha)) c_{-3+\alpha, 1+\beta} \\
& +a_{21}(2-(1+s)(-2+\alpha)) c_{-2+\alpha, \beta}
\end{aligned}
$$




$$
\begin{aligned}
& +b_{12} n(2-(1+s) \beta) c_{-2+\alpha, \beta} \\
& +a_{12} \sqrt{n}(1-(1+s)(-1+\alpha)) c_{-1+\alpha,-1+\beta} \\
& +b_{03} n^{3 / 2}(3-(1+s)(-1+\beta)) c_{-1+\alpha,-1+\beta} \\
& +b_{02} n(2-(1+s) \beta) c_{-1+\alpha, \beta} \\
& \left.-a_{03} n(1+s) \alpha c_{\alpha,-2+\beta}\right) \\
& \times((s+1) \alpha)^{-1}
\end{aligned}
$$

and, for any natural number $m, \omega_{m}$ is given by the following recursive formula:

$$
\begin{aligned}
\omega_{m}= & 2(1+s) c_{-3+m, 1} \\
& +\frac{1}{\sqrt{n}} a_{30}(3-(-2+m)(1+s)) c_{-2+m, 0} \\
& +b_{12} n(3+s) c_{-1+m,-1} \\
& +a_{21}(2-(-1+m)(1+s)) c_{-1+m,-1} \\
& +b_{03} n^{3 / 2}(3+2(1+s)) c_{m,-2} \\
& +a_{12} \sqrt{n}(1-m(1+s)) c_{m,-2}+b_{02} n(3+s) c_{m,-1} \\
& -a_{03}(1+m) n(1+s) c_{1+m,-3} .
\end{aligned}
$$

Applying Lemma 21 and computing with Mathematica, we have

$$
\begin{gathered}
\omega_{6}=-\frac{4 s-1}{\sqrt{n}} a_{30}, \quad \omega_{7} \sim 3(s+1) c_{03}, \\
\omega_{8} \sim-\frac{2}{5} \sqrt{n}(4 s-3)\left(a_{12}+3 n b_{03}\right), \\
\omega_{9} \sim-4 n^{5 / 2}(s-1) b_{02} b_{03} .
\end{gathered}
$$

From $\omega_{9}=0$, the analytic center problem of system (29) can be broken down into three cases: (1) $b_{02}=0$, (2) $b_{03}=0$, and (3) $s=1$.

Case 1. Consider $b_{02}=0$.

In this case, further calculation gives the following.

Theorem 22. For system (29), the first three quasi-Lyapunov constants at the origin are given by

$$
\begin{gathered}
\lambda_{1}=\frac{1}{\sqrt{n}} a_{30}, \quad \lambda_{2} \sim \frac{2}{5} \sqrt{n}\left(a_{12}+3 n b_{03}\right), \\
\lambda_{3} \sim \frac{4}{7} n^{3 / 2} b_{03}\left(a_{21}+n b_{12}\right) .
\end{gathered}
$$

In the above expression of $\lambda_{k}$, one has already let $\lambda_{1}=\lambda_{2}=$ $\cdots=\lambda_{k-1}=0, k=2,3$.

From Theorem 22, we obtain the following assertion.
Theorem 23. For system (29), all the quasi-Lyapunov constants at the origin are zero if and only if the first three quasiLyapunov constants at the origin are zero; that is, one of the following two conditions holds:

$$
\begin{gathered}
a_{30}=a_{12}=b_{02}=b_{03}=0 ; \\
a_{30}=b_{02}=0, \quad a_{12}+3 n b_{03}=0, \quad a_{21}+n b_{12}=0 .
\end{gathered}
$$

Relevantly, both conditions are the analytic center conditions of the origin.

Proof. When condition (35) is satisfied, system (29) can be brought to

$$
\begin{gathered}
\frac{d x}{d t}=y\left(1+a_{21} x^{2}+n a_{03} y^{2}\right), \\
\frac{d y}{d t}=-x\left(2 x^{2}-n b_{12} y^{2}\right),
\end{gathered}
$$

whose vector field is symmetric with respect to the origin.

When condition (36) is satisfied, system (29) can be brought to

$$
\begin{gathered}
\frac{d x}{d t}=y\left(1-n b_{12} x^{2}-3 n^{3 / 2} b_{03} x y+n a_{03} y^{2}\right), \\
\frac{d y}{d t}=-2 x^{3}+n b_{12} x y^{2}+n^{3 / 2} b_{03} y^{3},
\end{gathered}
$$

which is Hamiltonian and possesses the analytic first integral

$$
F_{1}(x, y)=x^{4}+y^{2}-n b_{12} x^{2} y^{2}-2 n^{3 / 2} b_{03} x y^{3}+\frac{1}{2} n a_{03} y^{4} .
$$

Case 2. Consider $b_{03}=0$.

In this case, $\lambda_{1}=\lambda_{2}=0$ yields that

$$
a_{30}=a_{12}=b_{03}=0 \text {. }
$$

Under this condition, system (29) becomes

$$
\begin{aligned}
& \frac{d x}{d t}=y\left(1+a_{21} x^{2}+n a_{03} y^{2}\right), \\
& \frac{d y}{d t}=-2 x^{3}+n b_{02} y^{2}+n b_{12} x y^{2},
\end{aligned}
$$

whose vector field is symmetric with respect to axis $x$. Thus, system (29) has an analytic center at the origin when condition (40) is satisfied.

Obviously, condition (35) is a special case of condition (40).

Case 3. Consder $s=1$.

In this case, we have the following. 
Proposition 24. For system (29), one can determine successively the terms of the formal series $M(x, y)=x^{4}+y^{2}+o\left(r^{4}\right)$, such that

$$
\begin{gathered}
\left(\frac{\partial X}{\partial x}+\frac{\partial Y}{\partial y}\right) M-2\left(\frac{\partial M}{\partial x} X+\frac{\partial M}{\partial y} Y\right) \\
=\sum_{m=1}^{7} \lambda_{m}\left[(2 m-5) x^{2 m+4}+o\left(r^{18}\right)\right],
\end{gathered}
$$

where $\lambda_{m}$ is the mth quasi-Lyapunov constant at the origin of system (29), $m=1,2, \ldots, 7$.

Further calculation gives the following.

Theorem 25. For system (29), the first seven quasi-Lyapunov constants at the origin are given by

$$
\begin{aligned}
& \lambda_{1}= \frac{1}{\sqrt{n}} a_{30}, \\
& \lambda_{2} \sim \frac{2}{5} \sqrt{n}\left(a_{12}+3 n b_{03}\right), \\
& \lambda_{3} \sim \frac{4}{35} n^{3 / 2} b_{03}\left(5 a_{21}+5 n b_{12}+11 n^{2} b_{02}^{2}\right), \\
& \lambda_{4} \sim-\frac{4}{7875} n^{9 / 2} b_{02}^{2} b_{03}\left(-1475 b_{12}+744 n b_{02}^{2}\right), \\
& \lambda_{5} \sim-\frac{8}{2512846875} n^{9 / 2} b_{02}^{2} b_{03} \\
& \times\left(589594375 a_{03}+395195814 n^{3} b_{02}^{4}\right), \\
& \lambda_{6} \sim-\frac{24}{4352606774140625} n^{13 / 2} b_{02}^{2} b_{03} \\
& \times\left(-1131416865765625 b_{03}^{2}+529893701720802 n^{3} b_{02}^{6}\right), \\
& \lambda_{7} \sim-\frac{10573115332617676917216}{38365073074138357421875} n^{23 / 2} b_{02}^{10} b_{03} .
\end{aligned}
$$

In the above expression of $\lambda_{k}$, we have already let $\lambda_{1}=\lambda_{2}=$ $\cdots=\lambda_{k-1}=0, k=2,3,4,5,6,7$.

$\lambda_{i}, i=1,2,3,4,5,6,7$, in expression (43) vanish if and only if one of conditions (36) and (40) holds.

Therefore, we see from Cases 1-3 the following.

Corollary 26. The origin of system (29) ((3)) is an analytic center if and only if condition (36) or (40) holds. to

Actually, when condition (36) holds, system (3) goes over

$$
\begin{gathered}
\frac{d x}{d t}=y^{2 n-1}\left(1-n b_{12} x^{2}-3 n b_{03} x y^{n}+a_{03} y^{2 n}\right), \\
\frac{d y}{d t}=-2 x^{3}+b_{12} x y^{2 n}+b_{03} y^{3 n}
\end{gathered}
$$

which is Hamiltonian and possesses the analytic first integral

$$
F_{2}(x, y)=x^{4}+\frac{1}{n} y^{2 n}-b_{12} x^{2} y^{2 n}-2 b_{03} x y^{3 n}+\frac{1}{2 n} a_{03} y^{4 n} .
$$

When condition (40) holds, system (3) goes over to

$$
\begin{gathered}
\frac{d x}{d t}=y^{2 n-1}\left(1+a_{21} x^{2}+a_{03} y^{2 n}\right), \\
\frac{d y}{d t}=-2 x^{3}+b_{02} y^{2 n}+b_{12} x y^{2 n},
\end{gathered}
$$

whose vector field is symmetric with respect to axis $x$.

\section{Multiple Bifurcation of Limit Cycles}

In this section, we are going to establish the conditions for $O(0,0)$ to be at most a weak focus of order seven, and, more, we will prove that the perturbed system of (29) can generate seven limit cycles enclosing an elementary node at the origin.

From the fact $\lambda_{1}=\lambda_{2}=\lambda_{3}=\lambda_{4}=\lambda_{5}=\lambda_{6}=0, \lambda_{7} \neq 0$, we obtain the following.

Theorem 27. For system (29), the origin is a weak focus of order seven if and only if

$$
\begin{gathered}
a_{30}=0, \quad a_{12}=-3 n b_{03}, \\
a_{21}=-\frac{1}{5} n\left(5 b_{12}+11 n b_{02}^{2}\right), \quad b_{12}=\frac{744}{1475} n b_{02}^{2}, \\
a_{03}=-\frac{395195814}{589594375} n^{3} b_{02}^{4}, \\
b_{03}^{2}=\frac{529893701720802}{1131416865765625} n^{3} b_{02}^{6}, \quad b_{02} b_{03} \neq 0 .
\end{gathered}
$$

Consider the perturbed system of (29):

$$
\begin{aligned}
\frac{d x}{d t}= & \delta(\varepsilon) x+y+\frac{1}{\sqrt{n}} a_{30}(\varepsilon) x^{3}+a_{21}(\varepsilon) x^{2} y \\
& +\sqrt{n} a_{12}(\varepsilon) x y^{2}+n a_{03}(\varepsilon) y^{3}, \\
\frac{d y}{d t}= & 2 \delta(\varepsilon) y-2 x^{3}+n b_{02} y^{2}+n b_{12}(\varepsilon) x y^{2} \\
& +n^{3 / 2} b_{03}(\varepsilon) y^{3} .
\end{aligned}
$$

In order to get seven limit cycles, we only need to show that, when condition (47) holds, the Jacobian of the function group $\left(\lambda_{1}, \lambda_{2}, \lambda_{3}, \lambda_{4}, \lambda_{5}, \lambda_{6}\right)$ with respect to the parameter group $\left(a_{30}, a_{12}, a_{21}, b_{12}, a_{03}, b_{03}\right)$ does not equal zero. A direct calculation gives that

$$
\begin{aligned}
& \left.\frac{\partial\left(\lambda_{1}, \lambda_{2}, \lambda_{3}, \lambda_{4}, \lambda_{5}, \lambda_{6}\right)}{\partial\left(a_{30}, a_{12}, a_{21}, b_{12}, a_{03}, b_{03}\right)}\right|_{(47)} \\
& =-\frac{127789436073926783638623356338176}{145260521069842113268585205078125} n^{23} b_{02}^{18} b_{03} \neq 0 .
\end{aligned}
$$

The above discussions indicate the following. 
Theorem 28. If the origin of system (29) ((3)) is a weak focus of order seven, for $0<\delta \ll 1$, making a small perturbation to the coefficient group $\left(a_{30}, a_{12}, a_{21}, b_{12}, a_{03}, b_{03}\right)$, then, for system (48), in a small neighborhood of the origin, there exist exactly seven small amplitude limit cycles enclosing the origin $\mathrm{O}(0,0)$, which is an elementary node.

Example 29. Take

$$
\begin{gathered}
\delta(\varepsilon)=\varepsilon^{56}, \quad a_{30}(\varepsilon)=-\varepsilon^{42}, \\
a_{12}(\varepsilon)=-\frac{9 \sqrt{58877077968978 / 20801689}}{7375} n^{5 / 2} c^{3} \operatorname{sign}(c) \\
-3 n \varepsilon^{2}+\varepsilon^{30}, \\
a_{21}(\varepsilon)=-\frac{3989}{1475} n^{2} c^{2}-n \varepsilon^{12}-\varepsilon^{20}, \\
b_{12}(\varepsilon)=\frac{744}{1475} n c^{2}+\varepsilon^{12}, \\
a_{03}(\varepsilon)=-\frac{395195814}{589594375} n^{3} c^{4}+\varepsilon^{6} \\
=\frac{3 \sqrt{58877077968978 / 20801689}}{7375} n^{3 / 2} c^{3} \operatorname{sign}(c)+\varepsilon^{2} \\
b_{02}=c \operatorname{sign}(c),
\end{gathered}
$$

where $c$ is an arbitrary nonzero real constant.

Straightforward computations by using Theorem 25 give the first seven quasi-Lyapunov constants at the origin of system (48):

$$
\begin{aligned}
& \lambda_{1}=-\frac{1}{\sqrt{n}} \varepsilon^{42}+o\left(\varepsilon^{42}\right), \\
& \lambda_{2} \sim \frac{2}{5} \sqrt{n} \varepsilon^{30}+o\left(\varepsilon^{30}\right)=0.4 \sqrt{n} \varepsilon^{30}+o\left(\varepsilon^{30}\right), \\
& \lambda_{3} \sim-\frac{12 \sqrt{58877077968978 / 20801689}}{51625} n^{3} c^{3} \operatorname{sign}(c) \varepsilon^{20} \\
& +o\left(\varepsilon^{20}\right) \approx-0.391061 n^{3} c^{3} \operatorname{sign}(c) \varepsilon^{20}+o\left(\varepsilon^{20}\right), \\
& \lambda_{4} \sim \frac{4 \sqrt{19625692656326 / 62405067}}{4375} n^{6} c^{5} \operatorname{sign}(c) \varepsilon^{12} \\
& +o\left(\varepsilon^{12}\right) \approx 0.512725 n^{6} c^{5} \operatorname{sign}(c) \varepsilon^{12}+o\left(\varepsilon^{12}\right), \\
& \lambda_{5} \sim-\frac{8 \sqrt{15955688129593038 / 76759}}{2839375} n^{6} c^{5} \operatorname{sign}(c) \varepsilon^{6} \\
& +o\left(\varepsilon^{6}\right) \approx-1.28458 n^{6} c^{5} \operatorname{sign}(c) \varepsilon^{6}+o\left(\varepsilon^{6}\right), \\
& \lambda_{6} \sim \frac{25434897682598496}{4352606774140625} n^{19 / 2} c^{8} \varepsilon^{2}+o\left(\varepsilon^{2}\right) \\
& \approx 5.8436 n^{19 / 2} c^{8} \varepsilon^{2}+o\left(\varepsilon^{2}\right)
\end{aligned}
$$

$$
\begin{aligned}
& \lambda_{7} \\
& \sim-\frac{31719345997853030751648 \sqrt{58877077968978 / 20801689}}{282942413921770385986328125} \\
& \quad \times n^{13} c^{13} \operatorname{sign}(c)+o(1) \approx-0.188604 n^{13} c^{13} \operatorname{sign}(c) \\
& \quad+o(1) .
\end{aligned}
$$

Then, for $0<\varepsilon \ll 1$, system (48) has seven limit cycles $\Gamma_{k}$ : $r=\tilde{r}\left(\theta, h_{k}(\varepsilon)\right)$ in a small neighborhood of the origin, where $h_{k}(\varepsilon)=O\left(\varepsilon^{k}\right), k=1,2,3,4,5,6,7$.

\section{Conflict of Interests}

The authors declare that they have no conflict of interests.

\section{Acknowledgments}

This work is supported in part by the National Nature Science Foundation of China (11101126 and 11261010) and the Scientific Research Foundation for Doctoral Scholars of HAUST (09001524).

\section{References}

[1] V. V. Amelkin, N. A. Lukashevich, and A. N. Sadovskii, Nonlinear Oscillations in the Second Order Systems, BGU Publications, Minsk, Belarus, 1982, (Russian).

[2] A. Andreev, "Investigation of the behaviour of the integral curves of a system of two differential equations in the neighbourhood of a singular point," Transactions of the American Mathematical Society, vol. 8, pp. 187-207, 1958.

[3] F. Takens, "Singularities of vector fields," Publications Mathématiques de l'Institut des Hautes Études Scientifiques, vol. 43, no. 1, pp. 47-100, 1974.

[4] R. I. Bogdanov, "Local orbital forms of vector fields on a plane," Trudy Seminara Imeni I. G. Petrovskogo, vol. 5, pp. 50-85, 1979.

[5] R. Moussu, "Symétrie et forme normale des centres et foyers dégénérés," Ergodic Theory and Dynamical Systems, vol. 2, no. 2, pp. 241-251, 1982.

[6] M. A. Teixeira and J. Yang, "The center-focus problem and reversibility," Journal of Differential Equations, vol. 174, no. 1, pp. 237-251, 2001.

[7] J. Giné, "Analytic integrability and characterization of centers for generalized nilpotent singular points," Applied Mathematics and Computation, vol. 148, no. 3, pp. 849-868, 2004.

[8] I. A. García and J. Giné, "Analytic nilpotent centers with analytic first integral," Nonlinear Analysis: Theory, Methods \& Applications, vol. 72, no. 9-10, pp. 3732-3738, 2010.

[9] H. Giacomini, J. Giné, and J. Llibre, "The problem of distinguishing between a center and a focus for nilpotent and degenerate analytic systems," Journal of Differential Equations, vol. 227, no. 2, pp. 406-426, 2006.

[10] Y. Liu and J. Li, "On third-order nilpotent critical points: integral factor method," International Journal of Bifurcation and Chaos in Applied Sciences and Engineering, vol. 21, no. 5, pp. 1293-1309, 2011.

[11] T. Liu, L. Wu, and F. Li, "Analytic center of nilpotent critical points," International Journal of Bifurcation and Chaos, vol. 22, no. 8, Article ID 1250198, 8 pages, 2012. 


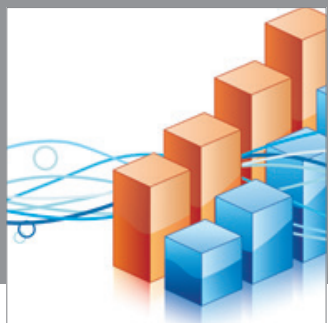

Advances in

Operations Research

mansans

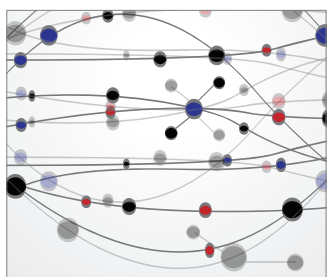

The Scientific World Journal
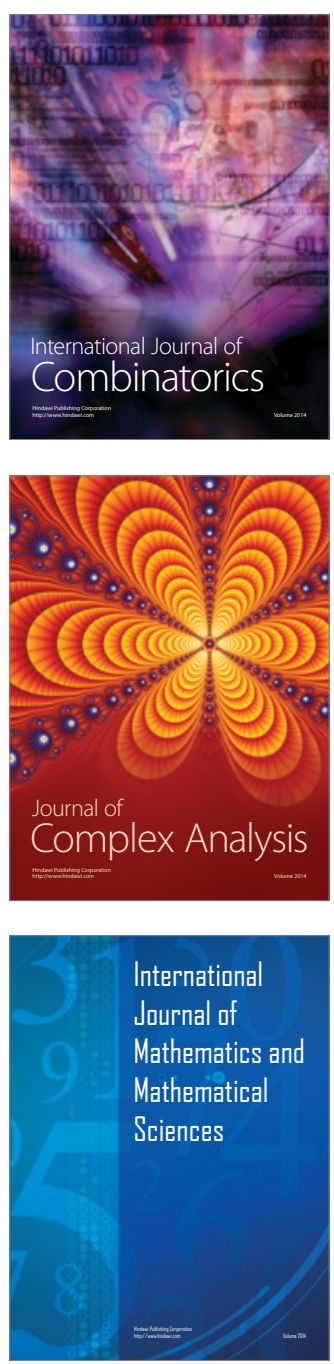
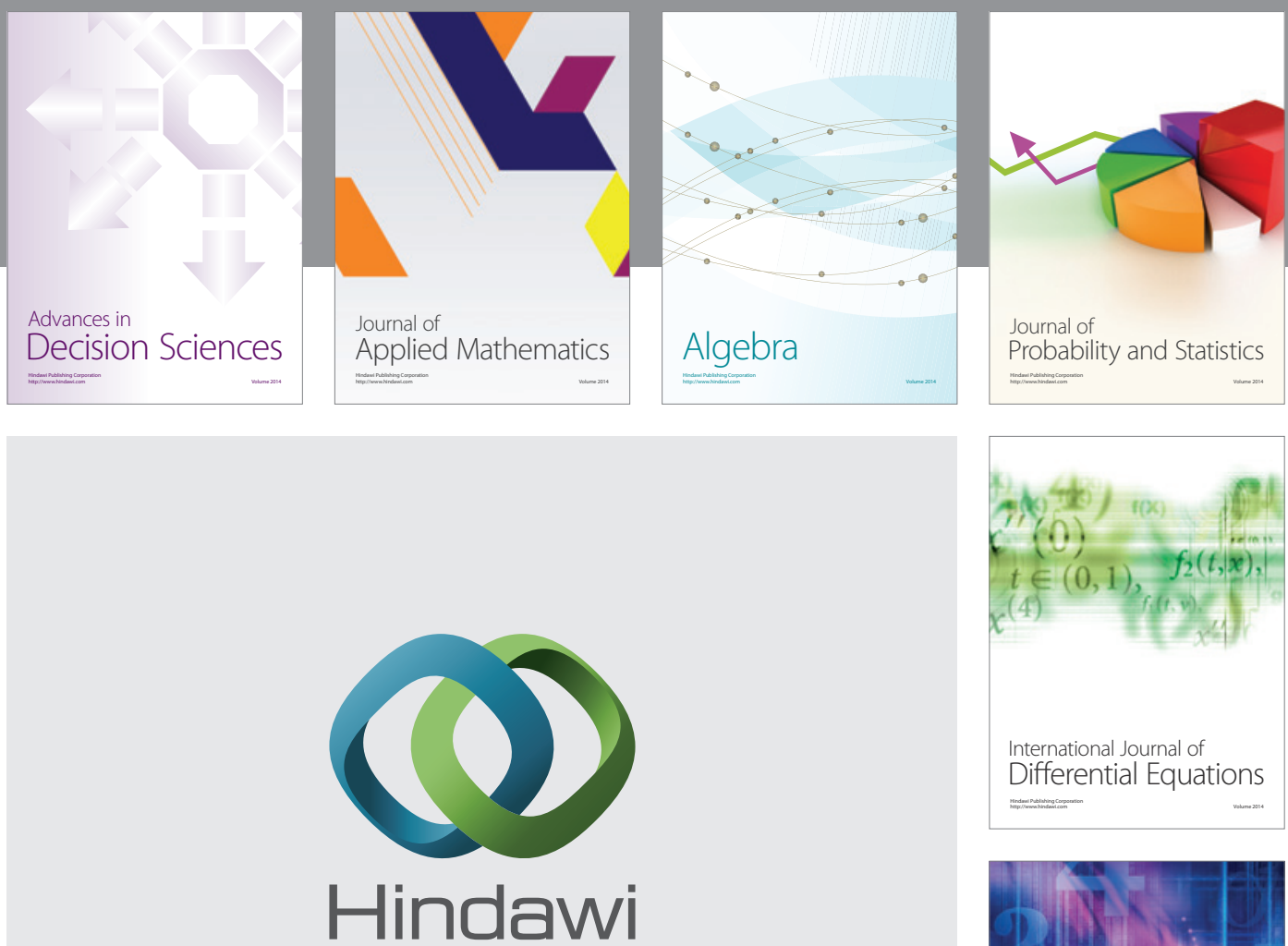

Submit your manuscripts at http://www.hindawi.com
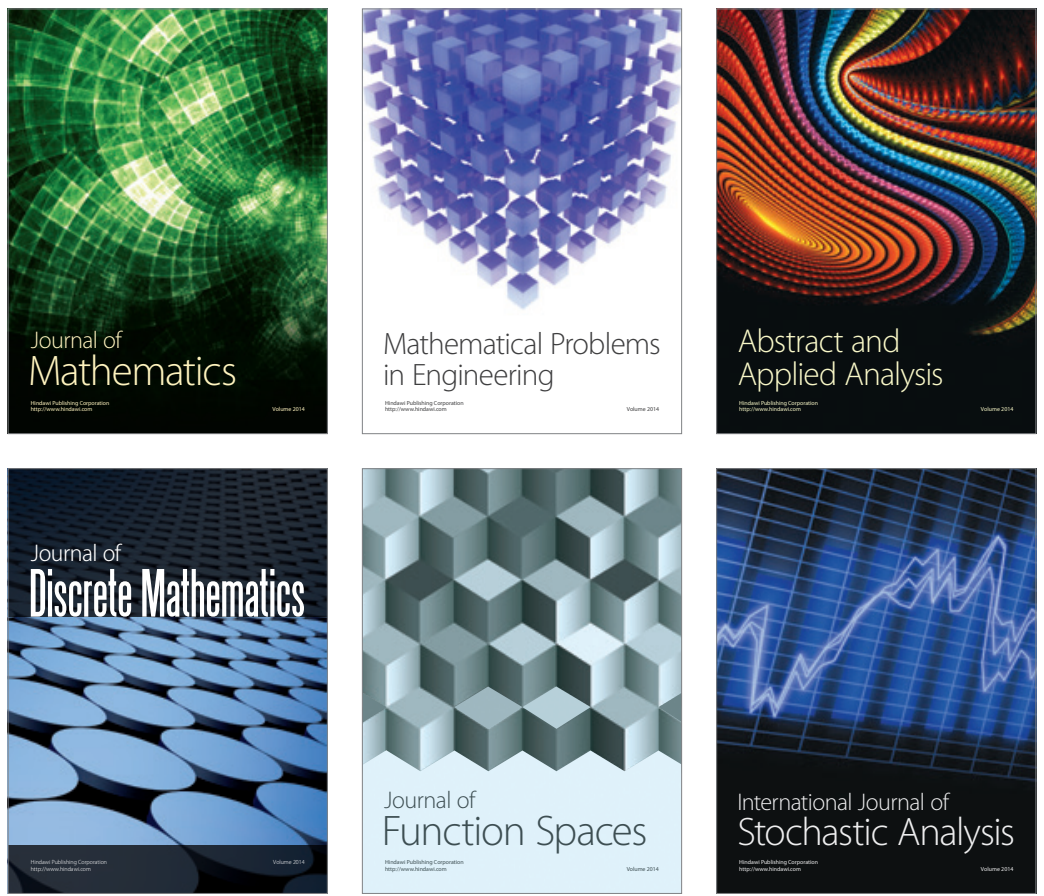

Journal of

Function Spaces

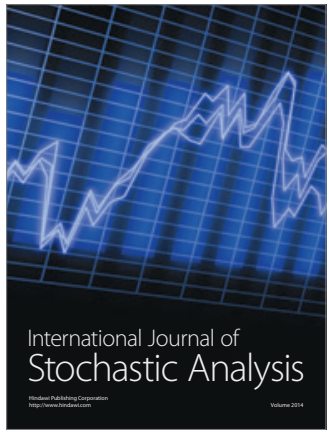

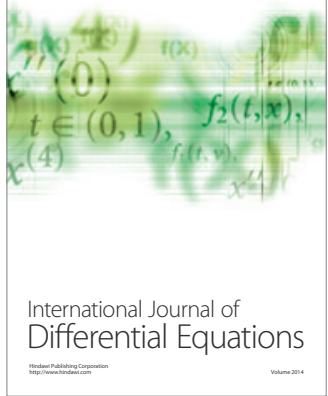
